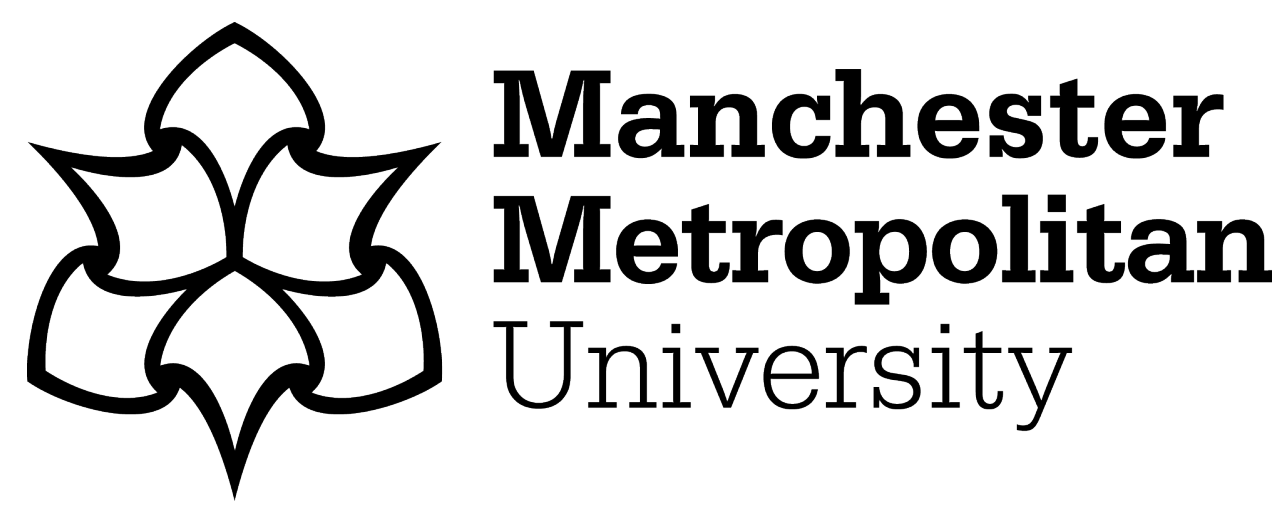

Kubie, L, King, LA ORCID logoORCID: https://orcid.org/0000-0002-07722378, Kern, ME, Murphy, JR, Kattel, S, Yang, Q, Stecher, JT, Rice, WD and Parkinson, BA (2017) Synthesis and Characterization of Ultrathin Silver Sulfide Nanoplatelets. ACS Nano, 11 (8). pp. 8471-8477. ISSN 1936-086X

Downloaded from: https://e-space.mmu.ac.uk/624324/

Version: Accepted Version

Publisher: American Chemical Society

DOI: https://doi.org/10.1021/acsnano.7b04280

Please cite the published version 


\title{
Synthesis and Characterization of Ultrathin Silver Sulfide Nanoplatelets
}

\author{
Lenore Kubie, ${ }^{\dagger}$ Laurie A. King, ${ }^{\dagger}$ Meghan E. Kern, ${ }^{\dagger}$ Joseph R. Murphy ${ }^{\ddagger}$ Subash

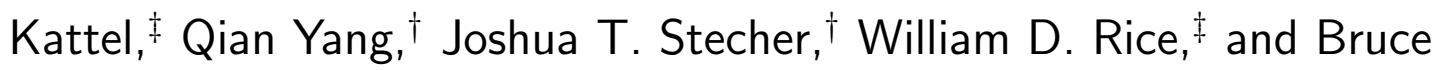 \\ A. Parkinson ${ }^{*} \dagger$ \\ $\dagger$ Department of Chemistry, University of Wyoming, Laramie, WY \\ $\ddagger$ Department of Physics, University of Wyoming, Laramie, WY \\ E-mail: bparkin1@uwyo.edu
}

\begin{abstract}
We report the synthesis of ultrathin, silver sulfide $\left(\mathrm{Ag}_{2} \mathrm{~S}\right)$ nanoplatelets (NPLs) synthesized via a one-pot method in ethylene glycol with 3-mercaptopropionic acid serving as both the sulfur precursor and platelet ligand. The colloidally synthesized nanoplatelets are exceptionally thin, with a thickness of only $3.5 \pm 0.2 \AA$, and a $1 \mathrm{~S}$ exciton Bohr diameter to confinement ratio of $\sim 12.6$. The NPL growth is shown to be quantized by layer thickness using absorption and photoluminescence (PL) spectroscopy. Transmission electron microscopy (TEM), atomic force microscopy (AFM), and x-ray diffraction analyses of the NPLs show that they correspond to the (202) plane of the $\beta-\mathrm{Ag}_{2} \mathrm{~S}$ structure. The PL quantum yield of these NPLs is $\sim 30 \%$ suggesting their potential use in biomedical imaging. Optoelectronic properties were evaluated via sensitized photocurrent spectroscopy with the resulting spectra closely matching the distinctive absorption spectral shape of the $\mathrm{Ag}_{2} \mathrm{~S}$ NPLs.
\end{abstract}




\section{Keywords}

nanoplatelet, silver sulfide, near-infrared emitter, photoluminescence quantum yield, incident photon conversion efficiency, colloidal synthesis

Semiconducting nanomaterials have attracted significant attention for their applications in both fundamental studies and opto-electronic applications including display technologies, light-emitting devices (LEDs) and photovoltaics. ${ }^{1-3}$ Two-dimensional (2D) semiconducting materials have garnered increased consideration in recent years due to their ultra-wide spectral absorption, ${ }^{4}$ spin-dependent transitions, ${ }^{5}$ high absorption coefficients, ${ }^{4,6,7}$ efficient charge transfer, ${ }^{8}$ and high detector photosensitivity. ${ }^{9}$ Moreover, given the small bandgaps of several NPL semiconductors (bulk $\beta-\mathrm{Ag}_{2} \mathrm{~S}$ has a band gap of $0.9 \mathrm{eV}^{10}$ ) and their high quantum confinement, multiple exciton generation (MEG, the formation of more than one exciton per incident absorbed photon) in the visible optical regime is likely, which would substantially increase photoconversion efficiencies. ${ }^{11}$ However, there has been limited work on the synthesis of semiconducting nanoplatelets (NPLs) especially in materials where the bulk structure is not layered. ${ }^{12-28}$ The lack of adaptability (e.g., inability to incorporate dopants), as well as the inherent difficulty in chemically producing high-quality 2D carbons and transition metal dichalcogenides, presents significant barriers to the technological implementation and widespread use of NPLs. However, NPLs produced via wet-chemical syntheses can be easily produced, manipulated, and scaled-up for applications.

In this Article, we report the preparation and characterization of colloidally synthesized, water-soluble, ultrathin $\mathrm{Ag}_{2} \mathrm{~S}$ NPLs. These NPLs are stable for several months in solution making them well suited for detailed optical and electrical studies. Atomic force microscopy (AFM), transmission electron microscopy (TEM), and x-ray diffraction (XRD) measure-

ments indicate that our $\mathrm{Ag}_{2} \mathrm{~S}$ NPLs have uniform thicknesses as small as $3.5 \pm 0.2 \AA$ and form along the (202) plane of the $\beta-\mathrm{Ag}_{2} \mathrm{~S}$ crystal structure. The sub-unit cell size of these NPLs creates a large quantum confinement ratio of over twelve times the $4.4 \mathrm{~nm}$ Bohr diameter of the $1 \mathrm{~S}$ exciton. ${ }^{29}$ As a consequence of this confinement, optical measurements 

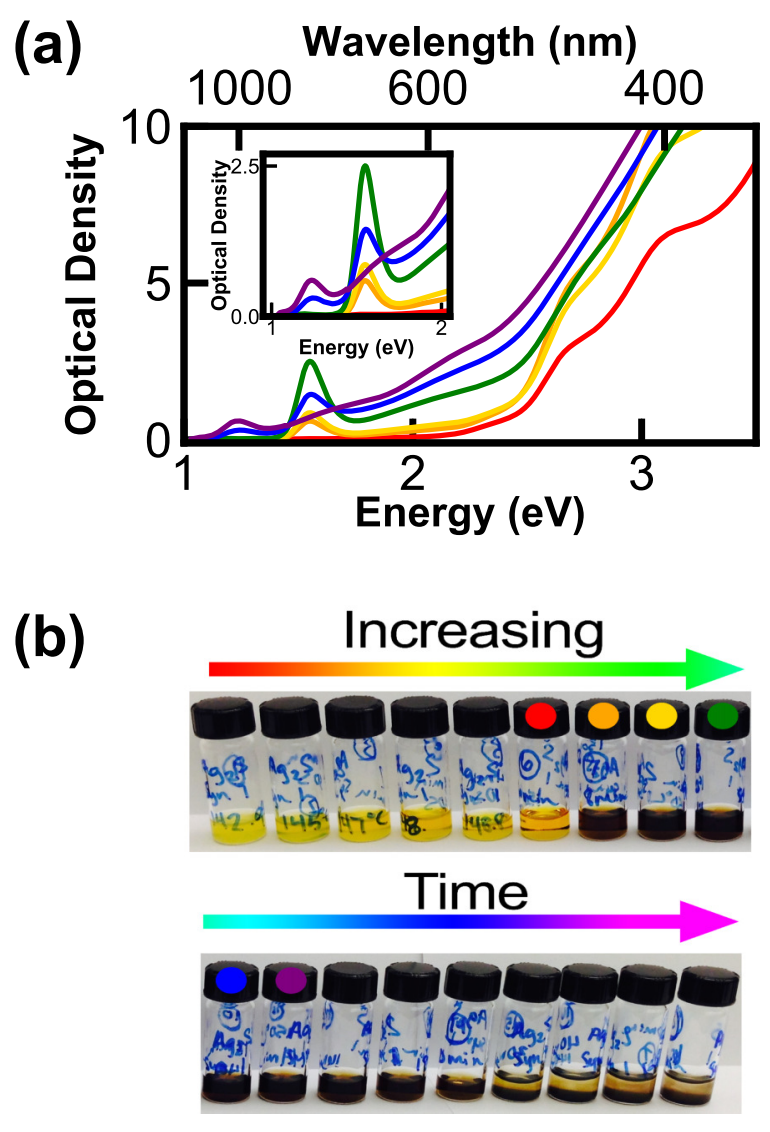

Figure 1: (a) Absorption spectra of $\mathrm{Ag}_{2} \mathrm{~S}$ NPLs during synthesis in ethylene glycol. Time proceeds from red to purple. At first there is an onset of absorbance at $\sim 2 \mathrm{eV}$ with shoulders at $\sim 2.7$ and $\sim 3.1 \mathrm{eV}$ (red). Over time, the $1.55 \mathrm{eV}$ exciton band grows in (orange to green) and then begins to disappear as another peak at $1.23 \mathrm{eV}$ begins to grow in (blue). Further growth increases the OD of the peak at $1.23 \mathrm{eV}$ (purple) until overgrowth causes flocculation of particles. Inset shows the exciton-region of the spectra enlarged. Solutions were diluted by a factor of ten, due to high absorptivity. The data was then multiplied by ten to present the true absorption. (b) Photograph of aliquots from the reaction over time. The precursor solution is cloudy white, but turns yellow upon slight heating. The solutions turn from yellow to brown as the reaction proceeds until all particles flocculate. Colored dots on the vial caps indicate the corresponding absorbance curve. UV-vis of aliquots before and after the aliquots marked in the photograph are dominated by scattering (not shown). 
of the as-synthesized NPLs reveal bright excitonic emission with a NIR-photoluminescence quantum yield $\left(\Phi_{\mathrm{PL}}\right)$ of $\sim 30 \%$, the highest ever measured from any form of $\mathrm{Ag}_{2} \mathrm{~S}$. Finally, we present sensitized photocurrent measurements, performed on the $\mathrm{Ag}_{2} \mathrm{~S}$ NPLs, showing the feasibility of this material for optical energy harvesting.

\section{Results and Discussion}

We monitored $\mathrm{Ag}_{2} \mathrm{~S}$ NPL syntheses as a function of time using optical absorption (Figure 1a). Four distinct stages, two of which show well-defined first excitonic band energies, are observed. The first stage of growth shows absorption shoulders at $\sim 2.7$ and $\sim 3.1 \mathrm{eV}$. Shortly thereafter, a well-defined absorption peak is measured at $1.55 \mathrm{eV}$ with an increase in the absorption baseline. The reaction mixture is visibly yellow during this growth stage. In the second growth stage, the mixture turns brownish in color (Figure 1b). During this reaction step, the intensity of the $1.55 \mathrm{eV}$ absorption band first increases and then begins to decrease. Concomitant with this drop in absorption at $1.55 \mathrm{eV}$, a new absorption band at $1.23 \mathrm{eV}$ appears. The third stage of growth is marked by a further increase of the $1.23 \mathrm{eV}$ absorption band followed by the loss of all features in the absorption spectrum as heating continues. In the fourth stage, the synthesis terminates when the particles flocculate. Significantly, the $\mathrm{Ag}_{2} \mathrm{~S}$ NPLs exhibit static excitonic energies, which is in contrast to colloidal quantum dot (QD) syntheses where the energy of the exciton gradually shifts to lower energies with increasing growth time. We attribute these discrete absorption peaks to NPLs with different numbers of two-dimensional layers since $2 \mathrm{D}$ confinement is not influenced by the lateral growth of the NPLs.

Imaging of the as-synthesized NPLs reveals that they are coated with a polymer or gellike material (TEM Figure S1 and AFM Figure S2). Although we have not been able to identify the material, ${ }^{13} \mathrm{C}$ and ${ }^{1} \mathrm{H}$ NMR of the unwashed reaction mixture suggests that it contains acetal-functionality (Figure S3). The height of the polymeric chains was deter- 
mined to be $0.2-1.0 \mathrm{~nm}$ via AFM measurements (Figure S2). The reaction to form this material is presumed to be catalyzed by the presence of silver, whether free or on the surface of the nanomaterials, or initiated by free-radicals generated when the sulfur precursor, 3-mercaptopropionic acid (3-MPA), reacts to produce sulfide.

Platelet heights were determined by AFM measurements of the washed NPLs deposited on mica(Figure 2c-f). Although the NPLs have significant variation in their lateral dimensions, the washed NPL heights are highly consistent. The AFM image of a washed NPL (exciton energy $=1.55 \mathrm{eV}$ ) and corresponding height trace (Figures 2) show that the washed NPL has a height of $\sim 3.5 \AA$, which correlates well to the smallest height possible with the $\mathrm{Ag}_{2} \mathrm{~S}$ platelets sandwiched by sulfur atoms (Figure 2b). Importantly, the proposed structure, seen in Figure 2b, maintains the bulk 2:1 Ag:S stoichiometry. This observation is not singular: AFM measurements of nearly a thousand washed NPLs, exhibiting the 1.55 $\mathrm{eV}$ absorption peak, show a mean height of $3.5 \AA$ (Figure $2 \mathrm{f}$ ). Consequently, we attribute the $1.55 \mathrm{eV}$ absorption feature to the formation of sub-nm, single-layer $\mathrm{Ag}_{2} \mathrm{~S}$ NPLs. The thicker platelets, associated with the exciton peak centered at $1.23 \mathrm{eV}$, appear to have a much broader height distribution, as seen in the red histogram in Figure 2f. As seen later, this broad distribution of NPL heights is due to the presence of different numbers of layers stacking along the [202] direction.

To confirm our proposed NPL layer structure, TEM micrographs were obtained from the washed $\mathrm{Ag}_{2} \mathrm{~S}$ NPLs (Figure 3). A TEM micrograph of the single-layer platelets (i.e., with an exciton peak at $1.55 \mathrm{eV}$ ) is shown in Figure 3a. The weak contrast with the background and the lack of visible NPL layering is consistent with imaging of a single-layer NPL. In contrast, Figure 2b shows a TEM image of a multi-layer NPL sample. Here, the different shaded regions in the image correspond to a stepwise increase in the number of $\mathrm{Ag}_{2} \mathrm{~S}$ layers. The NPL aggregation on the carbon TEM grids is not surprising, because of the bound polar 3-MPA ligands on the NPLs. It should be noted that the presence of bound 3-MPA has been confirmed via FTIR (Figure S5) and is supported by the $1.7 \pm 0.2 \mathrm{Ag}: \mathrm{S}$ ratio seen in EDS 
(a)
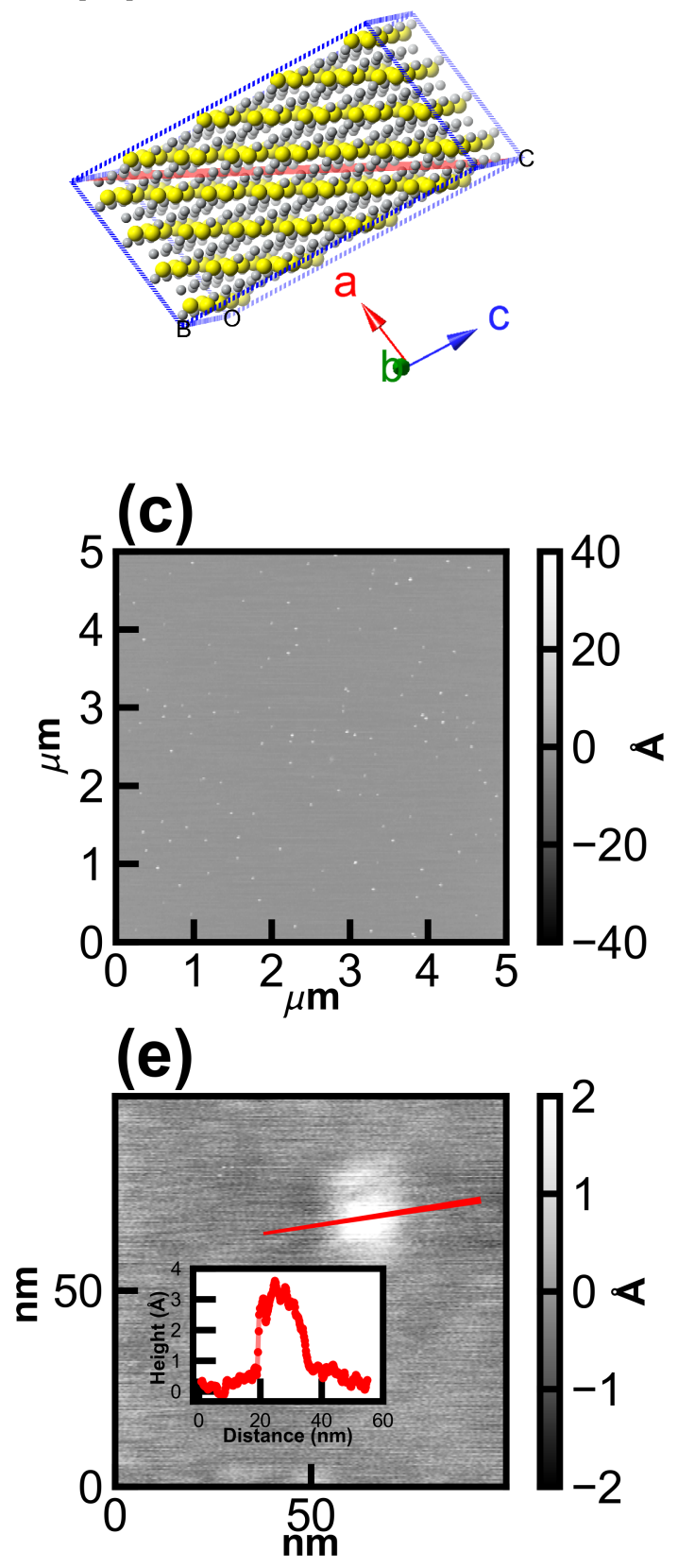

(b)
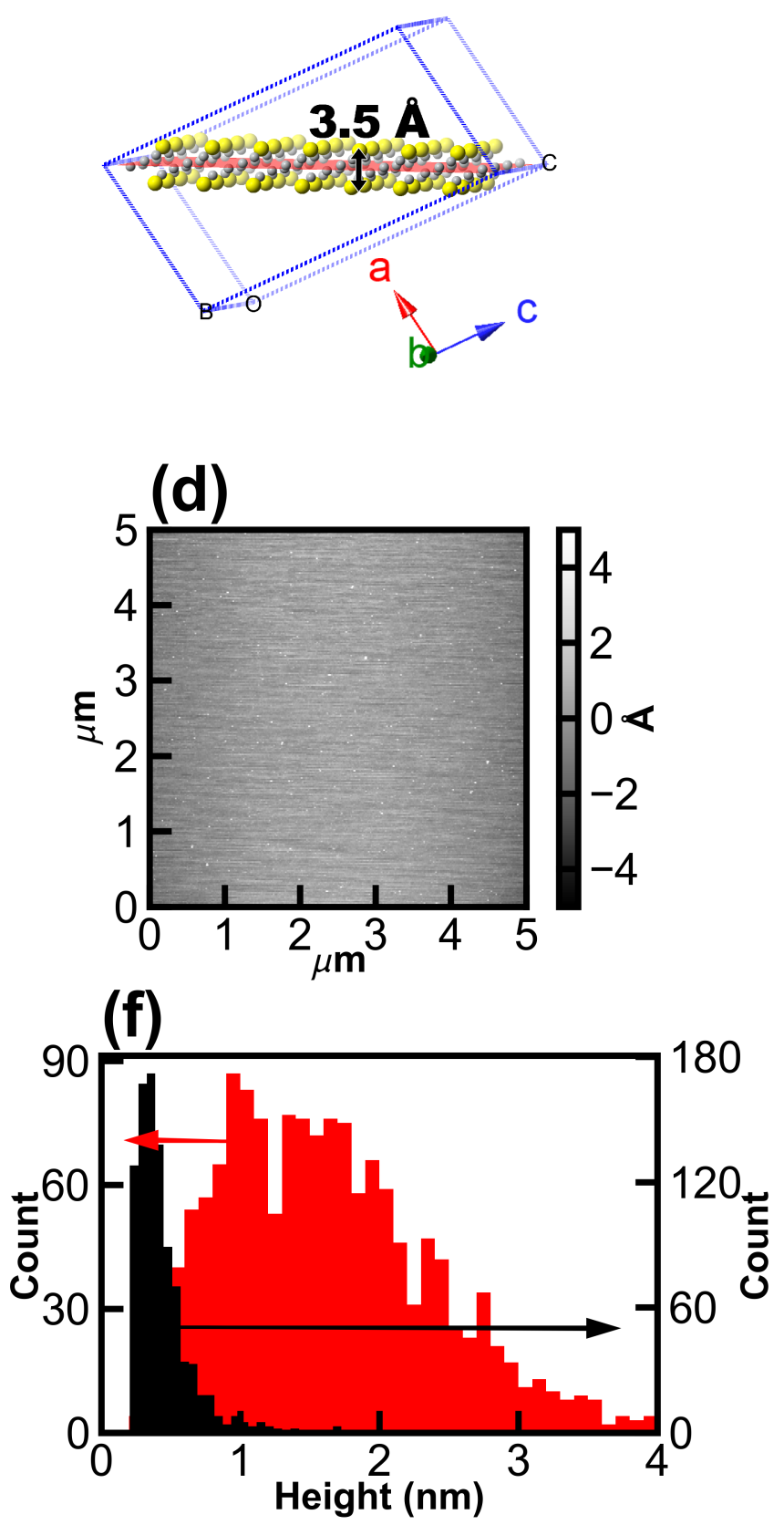

Figure 2: (a) $\beta-\mathrm{Ag}_{2} \mathrm{~S}$ bulk crystal structure. The (202) plane is shown in red. Sulfur atoms are shown in yellow and silver atoms are shown in gray. (b) Proposed platelet structure for nanoplatelets with an exciton band at $1.55 \mathrm{eV}$. The height of the plate is $3.5 \AA$. The extended unit cell is shown with a blue dotted line. Importantly, this structure maintains a 2:1 Ag:S stoichiometry. (c) AFM image of multi-layer $\mathrm{Ag}_{2} \mathrm{~S}$. (d) AFM image of single-layer $\mathrm{Ag}_{2} \mathrm{~S}$. (e) AFM image of a washed single-layer NPL. Red line shows the position of the height trace seen in the inset. (f) Histogram plot of the heights of the two NPL samples. The black bars represent the histogram for the sample with an exciton peak at $1.55 \mathrm{eV}(N=921)$, while the red bars represent the sample with the exciton peak at $1.23 \mathrm{eV}(N=1488)$. The bin size is $50 \mathrm{pm}$. 
(Figure S6). Because the ligand does not contribute to the height of the NPLs, but bound 3-MPA is present in thoroughly washed NPL samples, we surmise that the 3-MPA is bound to the exposed silver atoms around the edges of the NPLs.

In addition to providing support for the single- and multi-layer assignments, the TEM measurements also provide a direct estimate of the NPL lateral dimensions. As seen in Figures $3 \mathrm{a}$ and $\mathrm{b}$, the lateral NPL diameters fall between 5 and $20 \mathrm{~nm}$. Unlike the $3.5 \AA$ nanoplatelet heights, the estimated diameters are larger than the 1S exciton Bohr diameter of $4.4 \mathrm{~nm}$. Additionally, $126^{\circ}$ angles can be seen throughout Figures 3a-b, matching the angle between sulfur and silver atoms when the nanoplatelets are viewed from above (Figure S4). Given the measured NPL thickness, lateral dimensions, and $\mathrm{Ag}_{2} \mathrm{~S}$ exciton size, we are confident that these NPLs are sub-nanometer-thick 2D semiconductors.

Figure 3c shows direct TEM imaging of the NPL crystal lattice allowing for a very accurate measurement of the lattice spacing; this spacing is $2.7 \pm 0.2 \AA$, and matches the (022) Miller index. Notably, (022) runs nearly perpendicular to [202], and thus, is easily visible when the platelet is imaged from the top down. The TEM micrograph in Figure 3d shows what appear to be many edge-on stacked platelets with a spacing of $3.4 \pm 0.2 \AA$. The measured $3.4 \AA$ distance matches the spacing of the (202) Miller index of $\beta-\mathrm{Ag}_{2} \mathrm{~S}$ (Figure 4), and the height of the proposed nanoplatelet structure (Figure $2 \mathrm{~b}$ ). ${ }^{30}$

Powder x-ray diffraction (XRD) measurements (Figure 4) were obtained from washed $\mathrm{Ag}_{2} \mathrm{~S}$ NPLs, and are consistent with $\mathrm{Ag}_{2} \mathrm{~S}$ NPL formation. As seen in Figure 4, all of the peaks resolved in the XRD spectrum correlate well to peaks in the complex bulk $\operatorname{Ag}_{2} \mathrm{~S}$ pattern. Importantly, because of the sub-unit cell architecture of these NPLs across the [202] direction, only planes that run nearly perpendicular to [202] can be well resolved. Unlike bulk $\mathrm{Ag}_{2} \mathrm{~S}$, the NPL (101) peak is significantly broadened because the NPLs are only $\sim 3.5 \AA$ tall in the [202] direction. ${ }^{32}$ The XRD peaks corresponding to planes nearly perpendicular to [202] are also broadened, but not nearly as much as the (202) peak itself, further supporting the 2D structure of these NPLs. Additionally, the diffractogram clearly shows that the (022) 
(a)

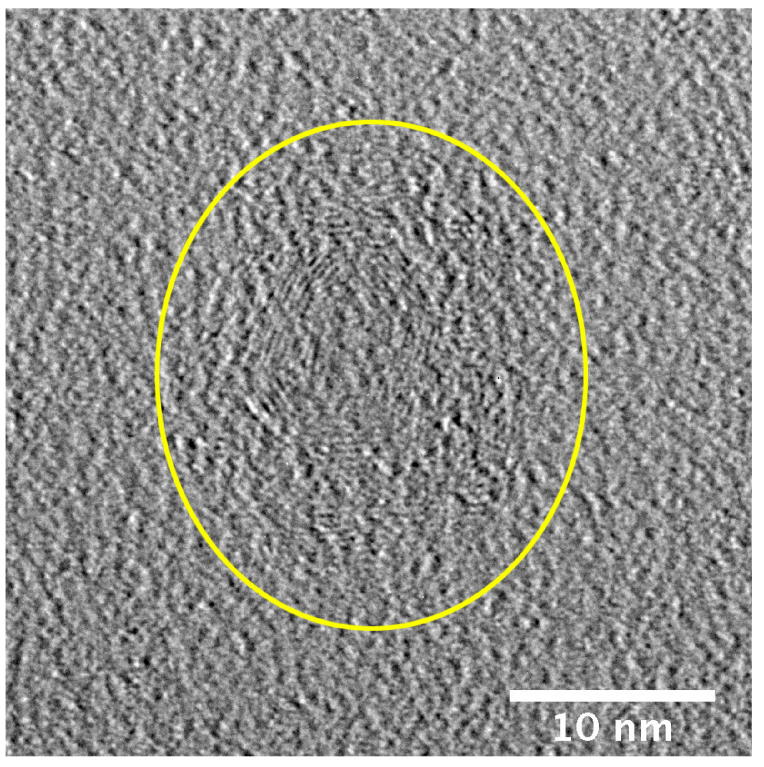

(c)

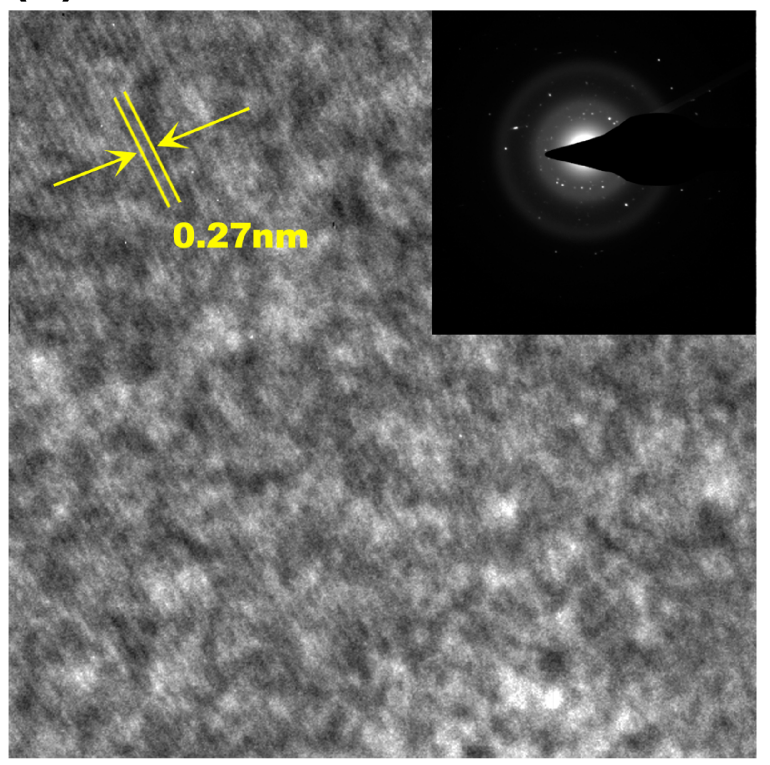

(b)

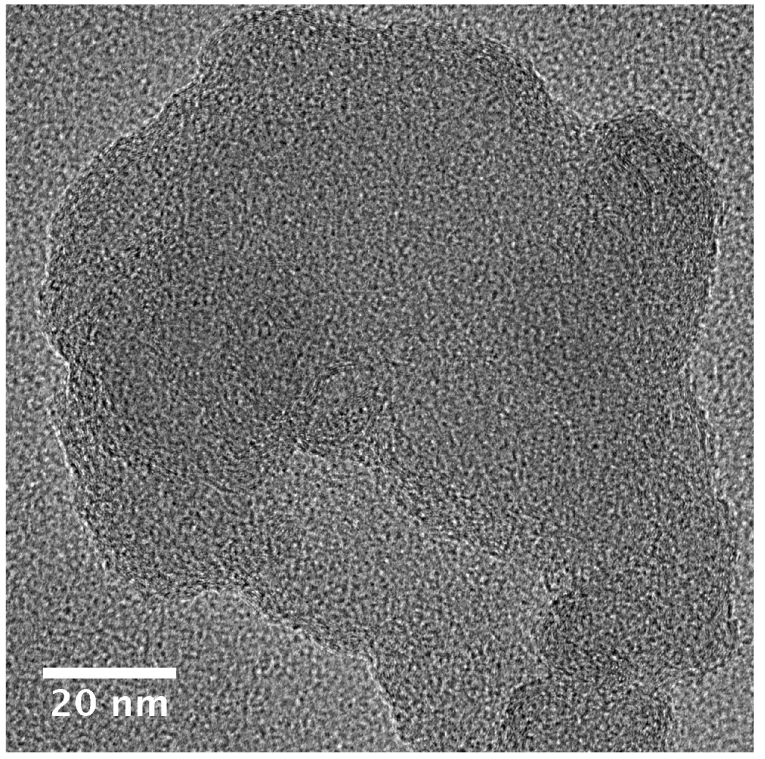

(d)

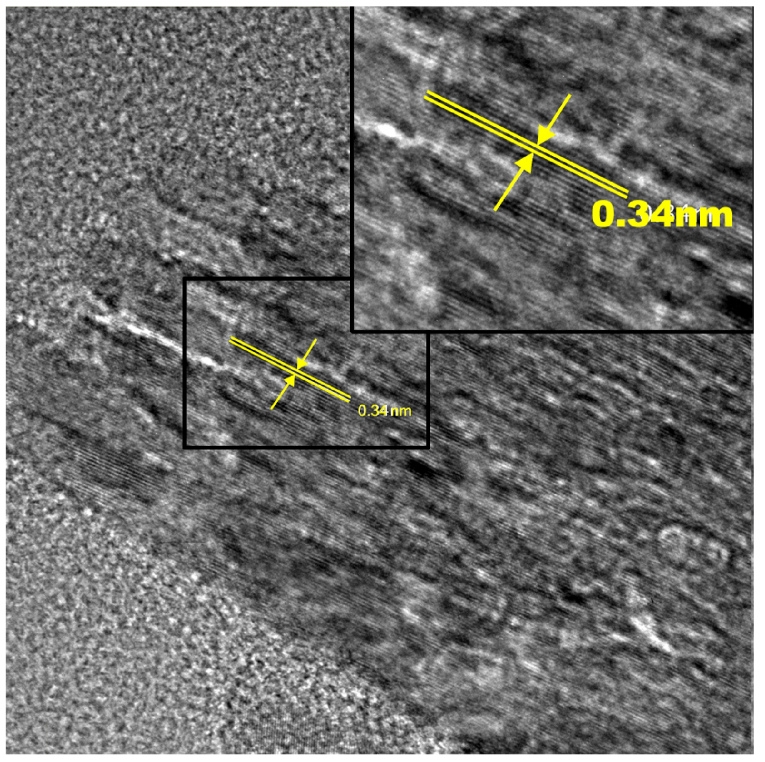

Figure 3: TEM micrographs of $\mathrm{Ag}_{2} \mathrm{~S}$ nanoplatelets. (a) Single-layer $\mathrm{Ag}_{2} \mathrm{~S}$ nanoplatelet. The lateral dimensions are $\sim 15 \mathrm{~nm}$. Yellow oval is loosely encircling the NPL to guide the eye because of the low contrast of a single-layer NPL. (b) Aggregated single- and multi-layer $\mathrm{Ag}_{2} \mathrm{~S}$ nanoplatelets. A stepwise increase in contrast between the carbon grid and nanoplatelet indicates an increase in the number of layers. (c) Single $\mathrm{Ag}_{2} \mathrm{~S}$ platelet showing $d$-spacing of $\sim 2.7 \AA$, which we assign to the (022) plane. This plane is also the most prominent peak seen in XRD (Figure 4), and runs perpendicular to [202] - the direction of the NPL. Inset shows the selected-area electron diffraction (SAED) from these platelets. (d) Many nanoplatelets stacked on top of one another with a spacing of $\sim 3.4 \AA$ (inset). 

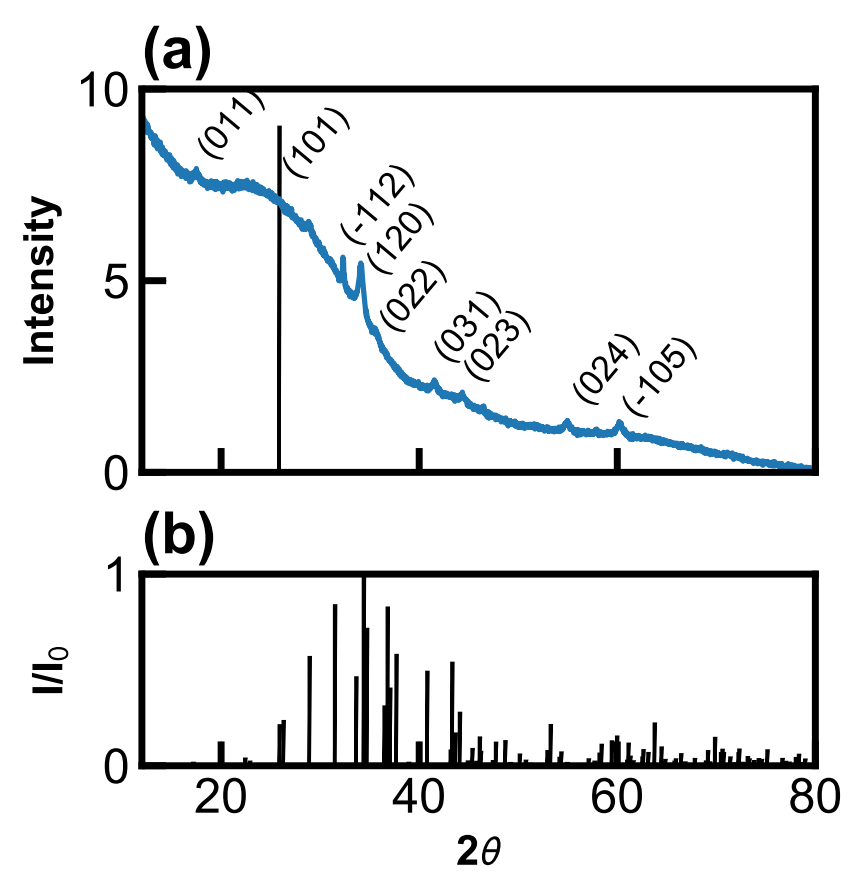

Figure 4: Top: XRD of $\mathrm{Ag}_{2} \mathrm{~S}$ NPLs with exciton peak located at $1.23 \mathrm{eV}$. The (101) peak, which is the plane the 2D nanoplatelet lies on, appears very broad, as would be expected for these structures. The vertical black line shows the position of the (101) peak in the bulk pattern. Only peaks corresponding to planes running nearly perpendicular to [202] can be seen, and proposed planes are labeled. Bottom: Known bulk $\mathrm{Ag}_{2} \mathrm{~S}$ XRD pattern. ${ }^{31}$ 
plane is the most prominent NPL XRD peak consistent with our lattice spacing estimates obtained by TEM.

The excitonic nature of the NPL absorption was confirmed using photoluminescence (PL) measurements of NPLs showing absorption peaks at $1.55 \mathrm{eV}$ and $1.23 \mathrm{eV}$ (Figure 5a). PL spectra with peaks at $1.45 \pm 0.05 \mathrm{eV}$ and $1.12 \pm 0.05 \mathrm{eV}$, respectively, were obtained using a $1.96 \mathrm{eV}(633 \mathrm{~nm})$ continuous-wave excitation source. Figure 5a shows that the excitonic emission from both NPL samples corresponds to a (global) Stokes shift of 100 meV. Both NPL spectra exhibit multi-featured emission lineshapes with a broad low-energy shoulder, which we attribute to surface-, edge-, and/or trap-state emission.

The importance of this 2D material is clearly demonstrated by the measured quantum yield, $\Phi_{\mathrm{PL}}$, of the near-infrared excitonic emission. Relative $\Phi_{\mathrm{PL}}$ measurements used the IR125 dye as a comparative emission standard. As seen in Figure 5b, the quantum yield for the $3.5 \AA$ NPLs is $\sim 30 \%$, which is much higher than previously synthesized $\mathrm{Ag}_{2} \mathrm{~S}$ nanomaterials (i.e., $\mathrm{Ag}_{2} \mathrm{~S}$ QDs), whose measured $\Phi_{\mathrm{PL}}$ reached $\sim 20 \% .{ }^{33}$ Although other inorganic materials, such as GaAs quantum wells, have much higher quantum yields $(80 \%$ at $\sim 1.5 \mathrm{eV}$ at low temperatures), these systems are not bio-compatible. Previous work has shown that both bulk $\mathrm{Ag}_{2} \mathrm{~S}$, and $\mathrm{Ag}_{2} \mathrm{~S}$ QDs are nontoxic, thus it is likely $\mathrm{Ag}_{2} \mathrm{~S}$ NPLs will also be nontoxic. ${ }^{34-36}$ $30 \%$ quantum yields in the near-infrared, coupled with improvements in their synthesis and photo-stabilization, may well make likely-nontoxic $\mathrm{Ag}_{2} \mathrm{~S}$ NPLs important players in nearinfrared optical and bio-related applications.

In order to improve $\Phi_{\mathrm{PL}}$, we examined how edge-passivation affects radiative emission efficiency. To examine this, $\mathrm{Ag}_{2} \mathrm{~S}$ NPLs were synthesized with 8-mercaptooctanoic acid (8MOA) in place of the 3-MPA used in our previous measurements. Because 8-MOA has a longer hydrocarbon-chain than 3-MPA, it should better isolate the edge states from solvent effects. Interestingly, despite these structural differences, $\Phi_{\mathrm{PL}}$ for both the 3-MPA-ligated and 8-MOA-ligated $\mathrm{Ag}_{2} \mathrm{~S}$ NPLs are not significantly different. This behavior suggests nonradiative recombination occurs on the faces of the NPLs, where no ligand is present. Because 
non-radiative recombination may be exacerbated at these surfaces when exposed to a solvent, further shielding of these NPLs may increase $\Phi_{\mathrm{PL}}$. At low intensities $\left(<0.05 \mathrm{~W} / \mathrm{cm}^{2}\right), \Phi_{\mathrm{PL}}$ does not significantly change, however as flux increases exciton-exciton annihilation processes dominate causing $\Phi_{\mathrm{PL}}$ to decrease (Figure 5b).

In addition to the optical and physical properties of these $\mathrm{Ag}_{2} \mathrm{~S}$ NPLs, we examined their optoelectronic properties. In this series of experiments, unwashed NPLs were adsorbed to the surface of natural tin dioxide $\left(\mathrm{SnO}_{2}\right)$ single crystals. Incident photon conversion efficiencies (IPCE) for photoexcited-electron injection were then measured as a function of wavelength and time (Figure 6). Prominent first excitonic peaks can be seen in Figure 6 confirming the appropriate band alignment between the $\mathrm{Ag}_{2} \mathrm{~S}$ and $\mathrm{SnO}_{2}$ band levels for electron injection into the $\mathrm{SnO}_{2}$ conduction band. Distinct exciton peaks were not present when the washed NPLs were used to sensitize $\mathrm{SnO}_{2}$, likely due to platelet aggregation on the surface (Figure S10). We observed a $30 \mathrm{~nm}(56 \mathrm{meV})$ red shift in the IPCE spectra relative to the $\mathrm{Ag}_{2} \mathrm{~S}$ NPL solution absorbance spectrum. Such shifts are often attributed to agglomeration of surface bound nanoparticles. ${ }^{37}$ It should be noted that the $56 \mathrm{meV}$ redshift is similar to those measured for QD sensitization of single-crystal $\mathrm{SnO}_{2}$; indeed, the shift observed for CdSe QD sensitized $\mathrm{SnO}_{2}$ is between 43 and $63 \mathrm{meV} .{ }^{38}$ Intriguingly, although there is a rapid decrease in photocurrent at the first excitonic band position, the photocurrent at higher energies $(>1.65 \mathrm{eV})$ is relatively stable. We speculate that the decay of photocurrent as a function of time may be due to rapid irreversible photooxidation of NPLs at their surfaces (analogous to the irreversible silver halide photooxidation seen in conventional photography) or the formation of thicker platelets. Similar results were obtained for systems sensitized with $\mathrm{Ag}_{2} \mathrm{~S}$ NPLs synthesized using thioglycolic acid (TGA) (Figure S11). Work on optimization of similar systems is ongoing. 


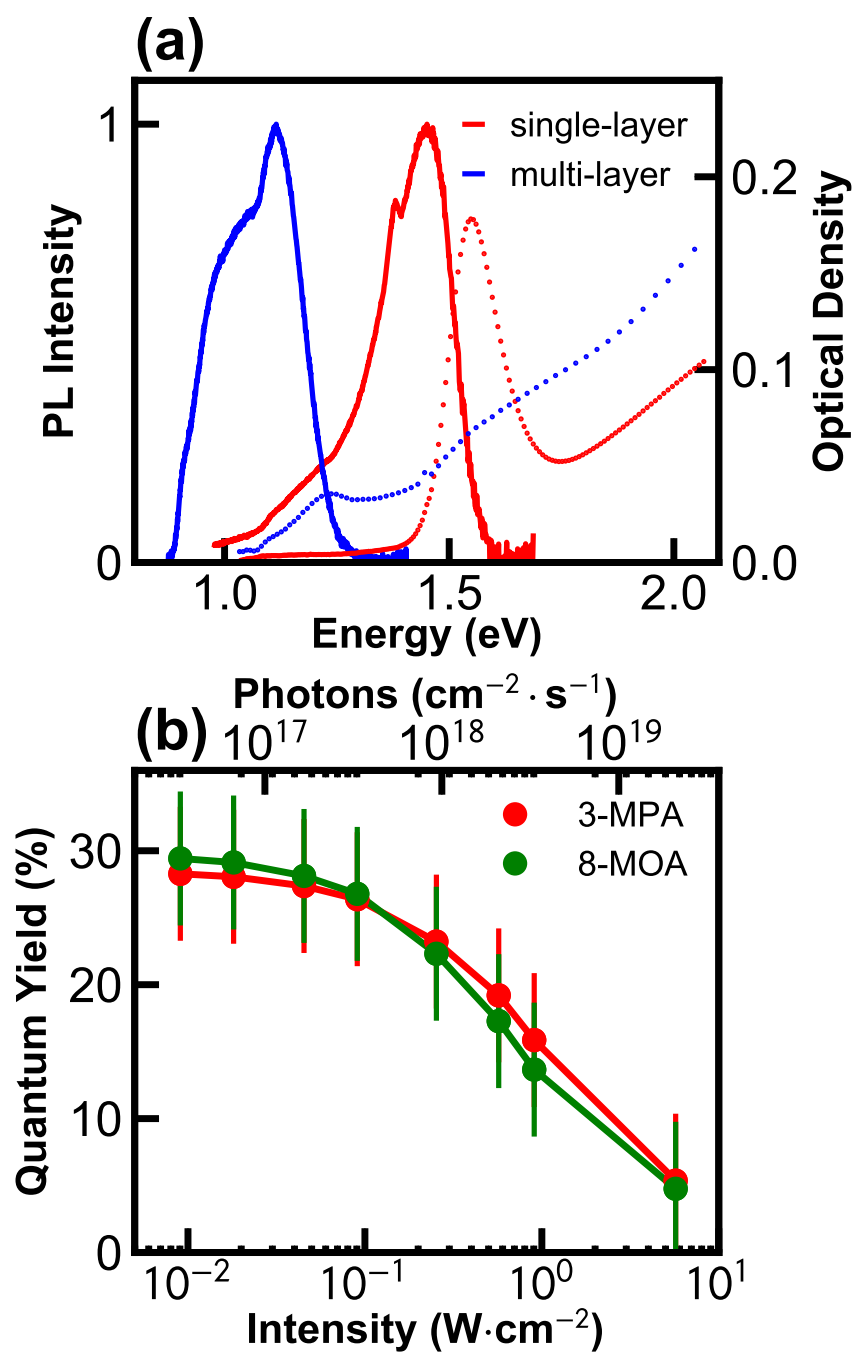

Figure 5: (a) Photoluminescence spectra of the two species of $\mathrm{Ag}_{2} \mathrm{~S}$ NPLs in water. Both samples were excited at $1.96 \mathrm{eV}$ and have trap and surface-state emission red-shifted from the PL maxima. The smaller $(3.5 \AA)$ platelets (with a $1.55 \mathrm{eV}$ exciton absorption; red dotted) have a emission maxima at $1.45 \pm 0.05 \mathrm{eV}$ (solid red). The multi-layer platelets (with a 1.23 $\mathrm{eV}$ exciton absorption; blue dotted) have a PL maxima of $1.12 \pm 0.05 \mathrm{eV}$ (blue solid). (b) Photoluminescence quantum yield of 3-MPA-ligated (red) and 8-MOA-ligated (green) $3.5 \AA$ $\mathrm{Ag}_{2} \mathrm{~S}$ NPLs as-prepared. Samples were excited with $1.70 \mathrm{eV}(730 \mathrm{~nm})$ light, and PL quantum yield values were measured across a range of intensities at the $1.45 \mathrm{eV}$ PL maximum. 

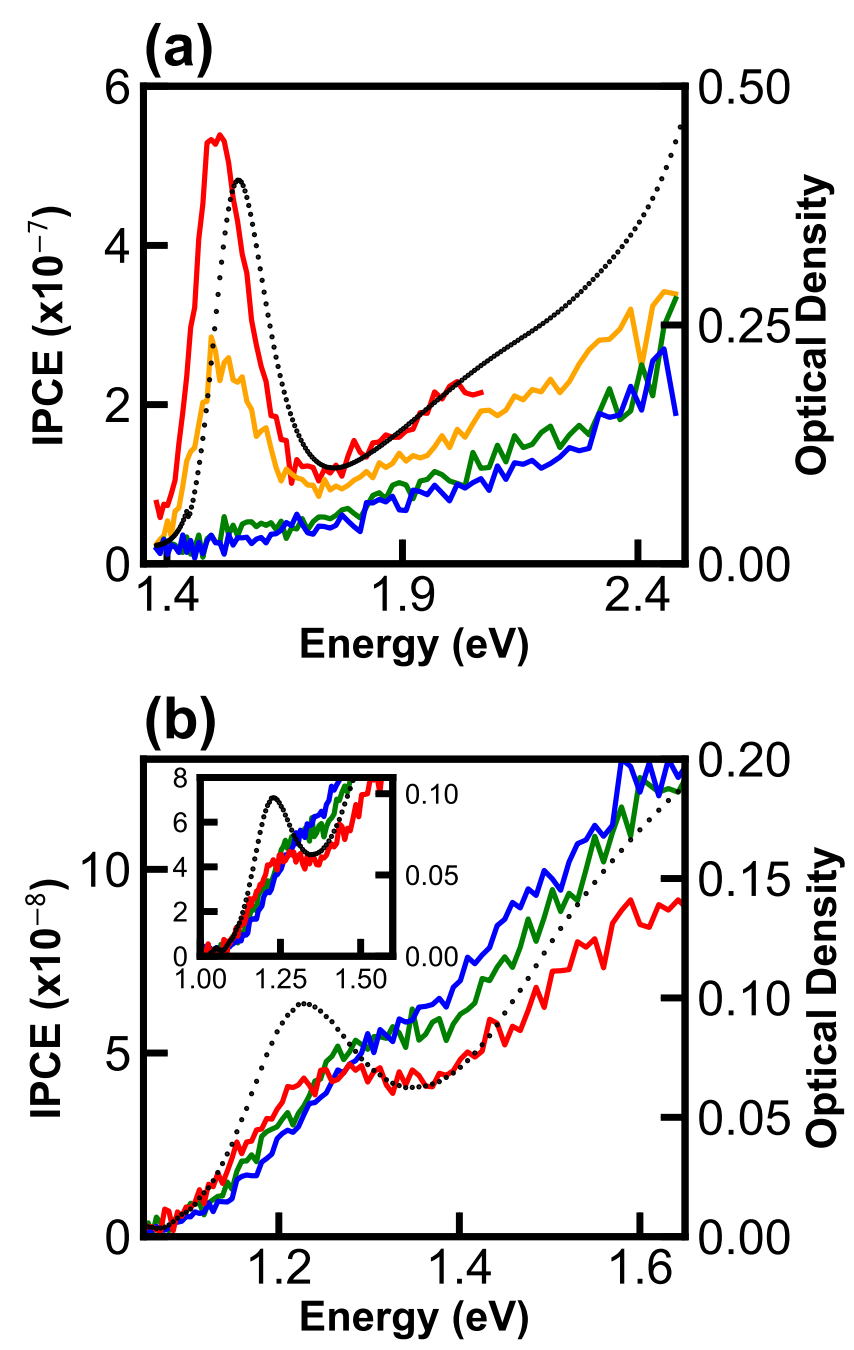

Figure 6: Photocurrent spectra for $\mathrm{Ag}_{2} \mathrm{~S}$ NPLs on $\mathrm{SnO}_{2}$ acquired with $0.5 \mathrm{mM}$ ferrocene in acetonitrile and a platinum wire counter electrode. Photocurrent spectra as a function of

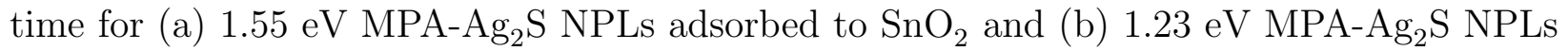
adsorbed to $\mathrm{SnO}_{2}$. Inset: Enlargement of the exciton peak region. Corresponding absorption spectra for $\mathrm{Ag}_{2} \mathrm{~S}$ NPLs overlaid as black dotted lines. Spectra were taken in direct succession with approximately 20 minutes in between, and time proceeds from red to blue. 


\section{Conclusions}

In summary, ultrathin semiconducting $\mathrm{Ag}_{2} \mathrm{~S}$ NPLs were produced using a one-pot synthesis. The nanoplatelets were characterized by both optical and physical means, and are shown via ICPE measurements to be a potential material for photoconversion applications. We have demonstrated that the synthesized NPLs presented here are $3.5 \pm 0.2 \AA$ tall, making them excellent candidates for fundamental studies of high-binding energy, two-dimensional excitons. ${ }^{12,14,17,18,20,21,24,25}$ Additionally, unlike other 2D materials (e.g. $\mathrm{MoS}_{2}$ and graphene), their colloidal synthesis should make both compositional- and ligand-manipulation relatively easy. The preference for platelet growth may be related to edge-sites being active for dissociation of the C-S bond of the MPA and/or poisoning of the (202) face by the polymeric reaction products. Previous reports utilizing similar synthetic methods have reported the synthesis of $\mathrm{Ag}_{2} \mathrm{~S}$ quantum dots of various sizes. ${ }^{33,35,39}$ We have observed that the $\mathrm{Ag}_{2} \mathrm{~S}$ NPLs will readily grow at room temperature if care is not taken to throughly wash to remove all precursors, and the eventual products do appear to be QDs in TEM micrographs (not shown). It is unclear if these studies mistakenly reported $\mathrm{Ag}_{2} \mathrm{~S}$ NPLs as $\mathrm{Ag}_{2} \mathrm{~S}$ QDs. These platelets are stable in solution for several months and therefore have a large potential for solution-based processing. Furthermore, the $\mathrm{Ag}_{2} \mathrm{~S}$ NPLs are exceptionally bright for a NIR-emitter $\left(\Phi_{\mathrm{PL}} \approx 30 \%\right)$. Additionally, the non-toxicity, and ease and relative low cost of synthesis make these materials attractive for additional photophysical studies and applications.

\section{Methods}

\section{$\mathrm{Ag}_{2} \mathrm{~S}$ Nanoplatelet Materials and Preparation.}

Materials. Silver nitrate (99.9999\% trace metal basis), 3-mercaptopropionic acid (99+\%), thioglycolic acid $(\geq 98 \%)$, and 8-mercaptooctinoic acid (95\%) were obtained from Aldrich. 
Tetrabutylammonium bromide (98+\%) was obtained from Alfa Aesar. Anhydrous ethylene glycol was obtained from Aldrich. Acetonitrile and acetone were obtained from various sources. DMSO was obtained from Fisher. DMSO-d6 was obtained from Cambridge Isotope Laboratories. IR-125 was obtained from Kodak. The $\mathrm{SnO}_{2}$ natural crystal was mined in Bolivia.

$\mathrm{Ag}_{2} \mathrm{~S}$ NPL Synthesis. $\mathrm{Ag}_{2} \mathrm{~S}$ NPL syntheses were developed with modifications to the procedure reported by Jiang et al. ${ }^{35}$ The synthesis employed 3-mercaptopropionic acid (MPA) as both the ligand and sulfur source. In brief, $\mathrm{AgNO}_{3} 0.043 \mathrm{~g}(0.25 \mathrm{mmol})$ was mixed with $45 \mu \mathrm{L} 3$-mercaptopropionic acid $(0.50 \mathrm{mmol})$ in $50 \mathrm{~mL}$ anhydrous ethylene glycol while purging with argon. The solution was brought to and held at $110{ }^{\circ} \mathrm{C}$ for 45 minutes using a heating mantle. The temperature was then increased to $130^{\circ} \mathrm{C}$ to promote particle growth. The procedure was also repeated with some modification using thioglycolic acid (TGA) and 8-mercaptooctanoic (MOA) acid as a sulfur precursor in place of MPA in the same molar ratio to silver. The synthetic procedure remained the same as that of MPA with the exception of a growth temperature of $180^{\circ} \mathrm{C}$ and $160^{\circ} \mathrm{C}$ respectively. All reactions were monitored via UV-Vis. Notably, the early aliquots of the reaction are more viscous, which we attribute to a more highly networked gel/polymer.

$\mathrm{Ag}_{2} \mathbf{S} \mathrm{UV}-\mathrm{Vis}$. As seen in Figure 1, $\mathrm{Ag}_{2} \mathrm{~S}$ NPL absorbance spectra were collected throughout the NPL synthesis to monitor the reaction progress. UV-vis spectroscopy was performed on a PerkinElmer Lambda 950 UV-Vis spectrometer. Solutions were diluted by ten-times for the measurement (because of the high optical density, OD). As such, the optical density spectra shown in Figure 1 were plotted at ten-times the measured absorbance to report the true OD of the raw solution.

$\mathrm{Ag}_{2} \mathrm{~S}$ washing. $7 \mathrm{~mL}$ of acetone was added to $3 \mathrm{~mL}$ of the resultant $\mathrm{Ag}_{2} \mathrm{~S}$ solution in a $15 \mathrm{~mL}$ disposable centrifuge tube. Unlike common QD purification procedures, the ethylene glycol-suspended NPLs required several hours to crash out. The solution was kept in the dark for several hours before precipitation occurred (generally $\sim 5$ hours). The cloudy solution 
was shaken briefly and then centrifuged for 15 minutes in an Adams physician's compact centrifuge $\left(\mathrm{RCF}_{\max }=924 \mathrm{~g}\right)$. After pellet formation, the supernatant was discarded and pellet was washed at least once more in acetone and then re-suspended in deionized water. Note, if the pellet would not resuspend readily, to enhance solubility several drops of $\sim 0.001 \mathrm{M}$ $\mathrm{NaOH}$ were added to the water to slightly increase the $\mathrm{pH}$ to above 8. If $\mathrm{NaOH}$ is added before the NPLs are throughly washed, the NPLs tend to grow.

\section{Characterization Results}

Transmission electron microscopy. TEM was performed using a FEI Tecnai G2 F20 $200 \mathrm{kV}$ TEM. TEM grids of the as-prepared samples were prepared by drop-casting the unwashed $\mathrm{Ag}_{2} \mathrm{~S}$ onto holey carbon film TEM grids, and then wicking away excess solution after several seconds. Dark field images of the unwashed samples were collected. Washed samples were prepared by drop-casting washed $\mathrm{Ag}_{2} \mathrm{~S}$ NPLs onto pure-carbon grids, and wicking away excess solution after twenty seconds.

X-ray diffraction. XRD experiments were performed on washed, dry NPL-powder. All measurements were performed on a Rigaku SmartLab Diffraction System operated with Cu $\mathrm{K} \alpha$ radiation source $(\lambda=1.54 \AA)$.

Nuclear Magnetic Resonance. Both ${ }^{1} \mathrm{H}$ and ${ }^{13} \mathrm{C}$ NMR spectra were obtained on the raw reaction mixture post-synthesis with $\sim 10 \% \mathrm{v} / \mathrm{v}$ DMSO-d6. For the ${ }^{1} \mathrm{H}$ NMR spectrum, 128 scans were collected with a one-second relaxtion delay and a sweep width of 40 ppm (Figure S3a). For the ${ }^{13} \mathrm{C}$ NMR spectrum 256 scans were taken with a sweep width of $301 \mathrm{ppm}$, and a two-second relation delay was used (Figure S3b). Both spectra were taken of the same sample with a Bruker Ascend $600 \mathrm{MHz}$ NMR.

FIIR. To remove any signal from solvents, washed, dried NPL-powder was used to perform Fourier transform infrared (FTIR) experiments. FTIR data was collected with a Perkin Elmer Spectrum Two FTIR with a PIKE Technologies GaldiATR. 100 spectra were averaged. Energy dispersive x-ray spectroscopy. EDS measurements were performed on a FEI 
Quanta 450 FEG with an Oxford Instruments EDS detector. The analyzed sample was a film of washed and dried $\mathrm{Ag}_{2} \mathrm{~S}$ NPLs on copper tape.

Atomic force microscopy. AFM was performed on an Asylum Cypher ES AFM. Freshly cleaved mica disks were used as a substrate for the washed $\mathrm{Ag}_{2} \mathrm{~S}$ NPLs. To prepare the substrate, several drops of the cleaned $\mathrm{Ag}_{2} \mathrm{~S}$ solutions were placed on the fresh mica surface. After twenty seconds, the excess solution was wicked away with a Kimwipeß and the disk was dried under nitrogen. Approximately 1000 platelets of each size $(1.55 \mathrm{eV}$ and 1.23 eV exciton peaks) were used to gather height statistics. The particle analysis automated functions were used with a 150 pm cutoff and $200 \mathrm{~nm}^{2}$ cutoff to eliminate noise.

\section{Photoluminescence and Quantum Yield Measurements}

Photoluminescence (PL) measurements shown in Figure 5a of washed $\mathrm{Ag}_{2} \mathrm{~S}$ NPLs in water were performed on a custom-built PL system. A $633 \mathrm{~nm}$ HeNe gas laser $(0.261 \mathrm{~mW}$ incident power) was used as the excitation source. PL was collected with a $25.4 \mathrm{~mm}$ lens, passed through a $780 \mathrm{~nm}$ long-pass filter, and sent to a $300 \mathrm{~mm}$-long spectrometer with a liquid nitrogen-cooled 1D InGaAs photodiode array (Princeton Instruments NIR PyLoN). The slit width on the spectrometer was set at $500 \mu \mathrm{m}$. PL spectra were composed of twenty averaged frames, each obtained with a 30 sec integration time. All spectra were corrected for the spectral response of the detector.

The relative PL quantum yield $\left(\Phi_{\mathrm{PL}}\right)$ data shown in Figure 5b in the paper were obtained using the dye Indocyanine Green (IR-125) in fresh dimethylsulfoxide (DMSO), with a correction factor associated with the difference in solvent index of refraction, as the reference. The $\mathrm{Ag}_{2} \mathrm{~S}$ and IR-125 solutions were measured in $10 \mathrm{~mm} \times 10 \mathrm{~mm}$ quartz cuvettes using a PerkinElmer Lambda $950 \mathrm{UV}$-Vis spectrometer for absorption measurements. For the corresponding PL measurements, the excitation source was a continuous-wave, wavelengthtunable Ti:Sapphire laser. Emitted light was collected and dispersed using a $300 \mathrm{~mm}$ spectrometer (Acton SpectraPro 300i) coupled with a liquid nitrogen-cooled InGaAs 1D de- 
tector array (Princeton Instruments PyLoN-IR 1024-1.7). Solutions of both the dye and nanoplatelets were diluted to obtain a maximum absorption of $<0.1$ at energies below that of the excitation wavelength (to limit reabsorption of the PL by the solutions). A spectral position that was reasonably flat was used as the excitation wavelength; for this measurement, a wavelength of $730 \mathrm{~nm}$ was selected. Photoluminescence measurements for both the sample and the reference were conducted under identical conditions as described by Würth et al. ${ }^{40}$

\section{Incident Photon Conversion Efficiency Measurements}

The same $\mathrm{SnO}_{2}$ crystal was used for all sensitization studies. The crystals were cleaned prior to use by sonication in water and ethanol.

To obtain photocurrent spectra, the clean natural $\mathrm{SnO}_{2}$ crystals were immersed in solutions of $\mathrm{Ag}_{2} \mathrm{~S}$ NPLs for two hours to functionalize the surface. To evaluate the $\mathrm{Ag}_{2} \mathrm{~S}$ NPLs for optoelectronic applications, we performed incident photon conversion efficiency (IPCE) measurements using a potentiostat (Princeton Applied Research EG\&G, 174A), lock-in amplifier (Stanford Research, SR830), and optical chopper (Stanford Research Systems SR540). A halogen lamp (Newport) in conjunction with a motorized monochromator (Jarrell-Ash Fischer Scientific) was used to produce the incident monochromatic light beam. This light was passed through a $450 \mathrm{~nm}$ long pass filter for the 500 to $900 \mathrm{~nm}$ measurement and a $600 \mathrm{~nm}$ long pass filter for the 800 to $1200 \mathrm{~nm}$ measurement. The light was subsequently focused with a focusing lens onto the NPL-sensitized crystal surface (front-side illumination).

\section{Acknowledgement}

The authors thank B. Durant for assistance with EDS and XRD measurements, and B. Leonard for some enlightening discussions. The authors wish to acknowledge the U.S. Department of Energy, Office of Science, Basic Energy Sciences, Division of Chemical Sciences 
for financial support through grant DE-SC0007115, and Geosciences and Biosciences and the University of Wyoming School of Energy Resources for financial support.

\section{Supporting Information Available}

TEM micrographs and AFM images of of unwashed $\mathrm{Ag}_{2} \mathrm{~S}$ NPLs, ${ }^{1} \mathrm{H}$ and ${ }^{13} \mathrm{C}$ NMR of gel/polymer side-product, top-down view of the model $\mathrm{Ag}_{2} \mathrm{~S}$ NPL, FTIR, EDS, AFM and additional TEM and IPCE measurements of NPLs. This material is available free of charge via the Internet at http://pubs.acs.org.

\section{References}

1. Bang, J. H.; Kamat, P. V. Quantum Dot Sensitized Solar Cells. A Tale of Two Semiconductor Nanocrystals: CdSe and CdTe. ACS Nano 2009, 3, 1467-1476.

2. Kim, M. R.; Ma, D. Quantum-dot-based Solar Cells: Recent Advances, Strategies, and Challenges. J. Phys. Chem. Lett. 2014, 6, 85-99.

3. Rogach, A. Quantum Dots Still Shining Strong 30 Years On. ACS Nano 2014, 8, 65116512 .

4. Nair, R. R.; Blake, P.; Grigorenko, A. N.; Novoselov, K. S.; Booth, T. J.; Stauber, T.; Peres, N. M. R.; Geim, A. K. Fine Structure Constant Defines Visual Transparency of Graphene. Science 2008, 320, 1308-1308.

5. Mak, K. F.; He, K.; Shan, J.; Heinz, T. F. Control of Valley Polarization in Monolayer $\mathrm{MoS}_{2}$ by Optical Helicity. Nat. Nanotechnol. 2012, 7, 494-498.

6. Barnham, K. W. J.; Ballard, I.; Connolly, J. P.; Ekins-Daukes, N. J.; Kluftinger, B. G.; Nelson, J.; Rohr, C. Quantum Well Solar Cells. Phys. E. 2002, 14, 27-36. 
7. Barnham, K.; Braun, B.; Nelson, J.; Paxman, M.; Button, C.; Roberts, J.; Foxon, C. Shortcircuit Current and Energy Efficiency Enhancement in a Lowdimensional Structure Photovoltaic Device. Appl. Phys. Lett. 1991, 59, 135-137.

8. Petoukhoff, C. E.; Krishna, M. B. M.; Voiry, D.; Bozkurt, I.; Deckoff-Jones, S.; Chhowalla, M.; O'Carroll, D. M.; Dani, K. M. Ultrafast Charge Transfer and Enhanced Absorption in $\mathrm{MoS}_{2}-$ Organic van der Waals Heterojunctions Using Plasmonic Metasurfaces. ACS Nano 2016, 10, 9899-9908.

9. Lopez-Sanchez, O.; Lembke, D.; Kayci, M.; Radenovic, A.; Kis, A. Ultrasensitive Photodetectors Based on Monolayer $\mathrm{MoS}_{2}$. Nat. Nanotechnol. 2013, 8, 497-501.

10. Junod, P. Relations Entre La Structure Cristalline Et Les Propriétés Électroniques Des Combinaisons $\mathrm{Ag}_{2} \mathrm{~S}, \mathrm{Ag}_{2} \mathrm{Se}, \mathrm{Cu}_{2} \mathrm{Se}$. Ph.D. thesis, 1959.

11. Nozik, A. J. Multiple Exciton Generation in Semiconductor Quantum Dots. Chem. Phys. Lett. 2008, 457, 3-11.

12. Chick, K. Y.; Nath, M.; Parkinson, B. TaS 2 Nanoplatelets Produced by Laser Ablation. J. Mater. Res 2006, 21, 1243-1247.

13. Fainblat, R.; Frohleiks, J.; Muckel, F.; Yu, J. H.; Yang, J.; Hyeon, T.; Bacher, G. Quantum Confinement-Controlled Exchange Coupling in Manganese (II)-Doped CdSe Two-dimensional Quantum Well Nanoribbons. Nano Lett. 2012, 12, 5311-5317.

14. Ithurria, S.; Dubertret, B. Quasi 2D Colloidal CdSe Platelets with Thicknesses Controlled at the Atomic Level. J. Am. Chem. Soc. 2008, 130, 16504-16505.

15. Ithurria, S.; Tessier, M. D.; Mahler, B.; Lobo, R. P. S. M.; Dubertret, B.; Efros, A. L. Colloidal Nanoplatelets with Two-Dimensional Electronic Structure. Nat. Mater. 2011, 10, 936-941. 
16. Jeong, S.; Yoo, D.; Jang, J.-t.; Kim, M.; Cheon, J. Well-defined Colloidal 2-D Layered Transition-Metal Chalcogenide Nanocrystals via Generalized Synthetic Protocols. J. Am. Chem. Soc. 2012, 134, 18233-18236.

17. Lauritsen, J. V.; Kibsgaard, J.; Helveg, S.; Topsøe, H.; Clausen, B. S.; Lægsgaard, E.; Besenbacher, F. Size-Dependent Structure of $\mathrm{MoS}_{2}$ Nanocrystals. Nat. Nanotechnol. 2007, 2, 53-58.

18. Mahler, B.; Nadal, B.; Bouet, C.; Patriarche, G.; Dubertret, B. Core/Shell Colloidal Semiconductor Nanoplatelets. J. Am. Chem. Soc. 2012, 134, 18591-18598.

19. Pedetti, S.; Nadal, B.; Lhuillier, E.; Mahler, B.; Bouet, C.; Abécassis, B.; Xu, X.; Dubertret, B. Optimized Synthesis of CdTe Nanoplatelets and Photoresponse of CdTe Nanoplatelets Films. Chem. Mater. 2013, 25, 2455-2462.

20. Schliehe, C.; Juarez, B. H.; Pelletier, M.; Jander, S.; Greshnykh, D.; Nagel, M.; Meyer, A.; Foerster, S.; Kornowski, A.; Klinke, C. Ultrathin PbS Sheets by TwoDimensional Oriented Attachment. Science 2010, 329, 550-553.

21. Sigman, M. B.; Ghezelbash, A.; Hanrath, T.; Saunders, A. E.; Lee, F.; Korgel, B. A. Solventless Synthesis of Monodisperse $\mathrm{Cu}_{2} \mathrm{~S}$ Nanorods, Nanodisks, and Nanoplatelets. J. Am. Chem. Soc. 2003, 125, 16050-16057.

22. Tang, Z.; Zhang, Z.; Wang, Y.; Glotzer, S. C.; Kotov, N. A. Self-Assembly of CdTe Nanocrystals into Free-Floating Sheets. Science 2006, 314, 274-278.

23. Tessier, M. D.; Spinicelli, P.; Dupont, D.; Patriarche, G.; Ithurria, S.; Dubertret, B. Efficient Exciton Concentrators Built From Colloidal Core/Crown CdSe/CdS Semiconductor Nanoplatelets. Nano Lett. 2013, 14, 207-213.

24. Wilcoxon, J.; Samara, G. Strong Quantum-Size Effects in a Layered Semiconductor: $\mathrm{MoS}_{2}$ Nanoclusters. Phys. Rev. B 1995, 51, 7299. 
25. Ye, C.; Bando, Y.; Shen, G.; Golberg, D. Thickness-Dependent Photocatalytic Performance of ZnO Nanoplatelets. J. Phys. Chem. B 2006, 110, 15146-15151.

26. Yu, J. H.; Liu, X.; Kweon, K. E.; Joo, J.; Park, J.; Ko, K.-T.; Lee, D. W.; Shen, S.; Tivakornsasithorn, K.; Son, J. S. Giant Zeeman Splitting in Nucleation-Controlled Doped CdSe: $\mathrm{Mn}^{2+}$ Quantum Nanoribbons. Nat. Mater. 2010, 9, 47-53.

27. Delikanli, S.; Akgul, M. Z.; Murphy, J. R.; Barman, B.; Tsai, Y.; Scrace, T.; Zhang, P.; Bozok, B.; Hernández-Martínez, P. L.; Christodoulides, J. Mn²+-Doped CdSe/CdS Core/Multishell Colloidal Quantum Wells Enabling Tunable Carrier-Dopant Exchange Interactions. ACS Nano 2015, 9, 12473-12479.

28. Jung, W.; Lee, S.; Yoo, D.; Jeong, S.; Miró, P.; Kuc, A.; Heine, T.; Cheon, J. Colloidal Synthesis of Single-Layer $\mathrm{MSe}_{2}(\mathrm{M}=\mathrm{Mo}, \mathrm{W})$ Nanosheets via Anisotropic Solution-Phase Growth Approach. J. Am. Chem. Soc. 2015, 137, 7266-7269.

29. Zhang, Y.; Liu, Y.; Li, C.; Chen, X.; Wang, Q. Controlled Synthesis of $\operatorname{Ag}_{2} \mathrm{~S}$ Quantum Dots and Experimental Determination of the Exciton Bohr Radius. J. Phys. Chem. C 2014, 118, 4918-4923.

30. Frueh, A. J. The Crystallography of Silver Sulfide, $\mathrm{Ag}_{2}$ S. Z. Kristallogr. 1958, 110, $136-144$.

31. Frueh, A. The Crystal Structure, Polymorphism and Twinning of Acanthite $\left(\mathrm{Ag}_{2} \mathrm{~S}\right)$. Acta Crystallogr. 1957, 10, 764-764.

32. Patterson, A. L. The Scherrer Formula for X-Ray Particle Size Determination. Phys. Rev. 1939, 56, 978-982.

33. Mir, W. J.; Swarnkar, A.; Sharma, R.; Katti, A.; Adarsh, K.; Nag, A. Origin of Unusual Excitonic Absorption and Emission from Colloidal $\mathrm{Ag}_{2} \mathrm{~S}$ Nanocrystals: Ultrafast Photophysics and Solar Cell. J. Phys. Chem. Lett. 2015, 6, 3915-3922. 
34. Zhang, Y.; Hong, G.; Zhang, Y.; Chen, G.; Li, F.; Dai, H.; Wang, Q. Ag 2 S Quantum Dot: A Bright and Biocompatible Fluorescent Nanoprobe in the Second Near-infrared Window. ACS Nano 2012, 6, 3695-3702.

35. Jiang, P.; Zhu, C.-N.; Zhang, Z.-L.; Tian, Z.-Q.; Pang, D.-W. Water-Soluble Ag $_{2}$ S Quantum Dots for Near-Infrared Fluorescence Imaging In Vivo. Biomaterials 2012, 33, 51305135.

36. Berthet, B.; Amiard, J.; Amiard-Triquet, C.; Martoja, M.; Jeantet, A. Y. Bioaccumulation, Toxicity and Physico-Chemical Speciation of Silver in Bivalve Molluscs: Ecotoxicological and Health Consequences. Sci. Total Environ. 1992, 125, 97-122.

37. Sambur, J. B.; Riha, S. C.; Choi, D.; Parkinson, B. Influence of Surface Chemistry on the Binding and Electronic Coupling of CdSe Quantum Dots to Single Crystal $\mathrm{TiO}_{2}$ Surfaces. Langmuir 2010, 26, 4839-4847.

38. King, L. A.; Yang, Q.; Grossett, M. L.; Galazka, Z.; Uecker, R.; Parkinson, B. A. Photosensitization of Natural and Synthetic $\mathrm{SnO}_{2}$ Single Crystals with Dyes and Quantum Dots. J. Phys. Chem. C 2016, 120, 15735-15742.

39. Sun, J.; Yu, W.; Usman, A.; Isimjan, T. T.; DGobbo, S.; Alarousu, E.; Takanabe, K.; Mohammed, O. F. Generation of Multiple Excitons in $\mathrm{Ag}_{2} \mathrm{~S}$ Quantum Dots: Single HighEnergy Versus Multiple-Photon Excitation. J. Phys. Chem. Lett. 2014, 5, 659-665.

40. Würth, C.; Grabolle, M.; Pauli, J.; Spieles, M.; Resch-Genger, U. Relative and Absolute Determination of Fluorescence Quantum Yields of Transparent Samples. Nat. Protoc. 2013, 8, 1535-1550. 